\title{
Study on Preparation and Application in Flocculants of Modified Lignin
}

\author{
Haiyin Liu \\ College of Life Science, Changchun Normal University \\ Changchun, Jilin 130022, China
}

\author{
Xiuyun Yang, Xiaoqiu Liu, Haibo Yao \& Yunhui Li (Corresponding author) \\ School of Chemistry and Environmental Engineering \\ Changchun University of Science and Technology, Changchun, Jilin 130022, China \\ E-mail: liyh@cust.edu.cn \\ Registration Code: Environmental Protection Agency of JiLin (2007-28)
}

\begin{abstract}
The lignin sulfonate was modified with olefins monomer by the method of radiation graft polymerization, by which a new type of natural polymer flocculants can be made. And results of the flocculation and sedimentation are tested in the furfural wastewater treatment process. Furfural wastewater is a complex composition, whose COD is about $20000 \mathrm{mg} / \mathrm{L}$, is difficult to be treated by conventional methods. Our results showed that the kind of flocculants can remove 50 percent $\mathrm{COD}$ or more from furfural wastewater after aerating and adjusting $\mathrm{pH}$ value to 9 .
\end{abstract}

Keywords: Lignin, Irradiation, Flocculants, Furfural wastewater

As a general plant of polymer, lignin is the main material to support plant growth, it constitute the cellulose with cellulose and hemicellulose together. Lignin in plants is the most abundant and most important organic polymer after cellulose. Lignin is non-toxic, source and rich in renewable resources, so it is widely used in industry. Generally, the commercial lignin is the derivative product from paper making by-products. Such as leather, dyestuff, food, architecture, and agricultural industries, it's mainly used as raw materials (such as mulan grain preparation) and additives (such as adhesives, dispersant, chelating agent and emulsifier) (Zhan HuaiYu. 2005).

Lignin, also can use as a flocculent flocculant modified lignin, due to several points such as the sources of the raw materials, inexpensive, non-toxic, easily biodegradable etc, and shows good application prospect. But there are some problems of low molecular weight and average less active absorption points, through the method of cross-linking reaction and condensation reaction, people want to change the spatial configuration and increase and molecular weight. Moreover, people try to introduce flocculation properties of functional and to improve the lignin flocculation properties further (Qiu XueQing, LouHongMing. 1999). Lignin can increase its quality of molecules after grafting in vinyl monomers; it's also a method to improve lignin's flocculation. Qianjun Liu etc(Liu Qianjun, ZhanHuaiYu, Liu mengru \& WuHong. 2003). Use potassium sulfate as the initiator and acryl amide monomer to preparate the decoloring agents lignin flocculation. GuiZhen Fang etc(Fang GuiZhen, HeWeiHua \& Song ZhanQian. 2003). Synthesize flocculant - lignin salt. B. Phillips (R. B. Phillips, W. Brown \& V. Stannett. 1973; R. B. Phillips, W. Brown \& V. Stannett. 1972) was studied the irradiation graft copolymerization reaction between styrene and lignin.. These researches contributed to the application of the lignin flocculant.

Base on the past research, this paper use lignosulphonate salt as photo base, join olefins grafted monomer, using irradiation graft polymerization method to preparate natural polymer flocculant lignin. This method is characteristic by the reaction process doesn't need solvent; the reaction process doesn't attract small molecules, so the process of using this kind of flocculant will not cause secondary pollution. We use the flocculant to deal with the furfural wastewater by flocculating sedimentation. Mainly because of COD is about $20000 \mathrm{mg} / \mathrm{L}, \mathrm{pH}<2$. According to the characteristics of furfural wastewater, we design the corresponding process. Practice has proved, in appropriate conditions, can achieve very good result flocculation.

\section{Experimental}

\subsection{Raw material and equipment}

(1)Llignosulphonate sodium/Beijing; technology Co., LTD. Beijing plant acid, Acetone Beijing chemical plants, Beijing olefin plant monomer.

(2)Furfural wastewater quality

Tan appearance, including a solid aerosol particle

COD $23025 \mathrm{mg} / \mathrm{L}$

$\mathrm{pH} \quad 4$ 
(3)Rotating evaporator (RE-52AA) Shanghai vibration; the laboratory equipment Co., LTD. Circulating water pump (SHB - multi-purpose type III) trading Co., LTD. Of Zhengzhou wall, 60Co emitter, Chinese academy of sciences, Changchun institute of applied chemistry.

\subsection{Flocculant preparation methods}

$300 \mathrm{ml}$ acetic acid was dropped into $500 \mathrm{ml}$ flask. drop $150 \mathrm{~g}$ lignosulphonate sodium into the flask slowly and group by group, of partial, then mix the lignin to be soluble by glass rod. put the flask of lignosulphonate mixed with acetic down for 10 minutes, and then put the filtered solid material into the flask, add appropriate acetone, and the do twice suction filter. We can get purificated yellow lignosulphonate after dry. According to the proportion of purification weigh

of lignosulphonate salt and olefin monomer, grinding, mixing, controlling conditions for radiation graft polymerization reaction which use $60 \mathrm{Co}$ as radiant point.

\subsection{Furfural wastewater flocculating sedimentation}

Taking fresh water samples from furfural, aerating, adding flocculants and auxiliary sorbents according to the design scheme, mixing fastly for $1 \mathrm{~min}$, stilling for $10 \mathrm{~min}$, getting the supernatant and calculating COD as well as COD removal.

\subsection{Flocculant surface morphology analysis}

We adopt the Nano - I atomic force microscope (type: S - favor - 0000-1) from American Schmidt Co to scan the water.

\subsection{Flocculants structure characterization}

We adopt the UV - 1240 type uv-vis spectrophotometer from Japanese to do the ultraviolet spectroscopic analysis.

\subsection{Furfural COD value method}

We adopt HACH COD tester (DR - 2010) from American, the temperature is $150^{\circ} \mathrm{C}$, dispelling time is $2 \mathrm{~h}$..

\section{Results and Discussion}

\subsection{Flocculant surface morphology analysis}

We should make the sample to $0.02 \mathrm{~g} / \mathrm{L}$ solution and spin coat on the slides about $1500 \mathrm{r} / \mathrm{min}$ in room temperature, In order to dry it, we observe the surface morphology in the atomic force microscope. Lignosulphonate salt is a three-dimensional granular structure of organic polymer, there's no organic polymer chain in its molecular structure. We can see from figure 2.1, sodium lignosulphonate is flat, $32.01 \mathrm{~nm}$ to devide; the surface properties of modified sodium lignosulphonate is changed. There are some obviously uniform and divide $46.32 \mathrm{~nm}$ to rise. This is because of in the modified lignin, the grafted surface by - C - C - composed of amorphous polymer composition of linear low molecular characteristics, main chain molecules, flexibility and good symmetry. When there-dimensional structure lignosulphonate salt on grafting, flexible chain on the surface properties of sodium lignosulphonate produces more changes, grafted long -chain formation obviously uplift and excluded in polarity membrane surface.

\section{2 grafted lignin up spectral analysis}

Lignin was typical of aromatic compounds. Aromatic compounds have characteristics of ultraviolet absorption, $280 \mathrm{~nm}$ will appear a stable absorption peak when there is aromatic substituent in lignin structure. Figure 2.2 shows ultraviolet absorption spectrum lines of lignosulphonate salt and modified lignin sulfonate olefin monomers.

The graph shows that lignin has obvious absorption peaks around $205 \mathrm{~nm}$ and $280 \mathrm{~nm}$ of ultraviolet spectrum. The monomer which is grafted to the lignin has been saturation, it lose phenacylmethylene groups to become substituent and there is greater space a resistance. A resistance can make the substituent which conjugate with benzene originally to be rejected out of benzene plane, and resistance also can not make substituent electrons overlap completely with benzene PI system, thus affecting the spectrum. Wave of benzene and the intensity of absorption with wave depend on the planar angle between substituent and benzene. After adjacent bits replace, because of the resistance increases, the factors make Angle reduced, cause absorption electronic overlap with strength successive reduced. Substituent produced blue affect in $280 \mathrm{~nm}$. The scope of absorb with strength reduction depends on how long and how much is grafting long chain. Modified sodium lignosulphonate.

\subsection{Liquid $\mathrm{pH}$ value of furfural COD removal rate}

In the Flocculating sedimentation furfural wastewater determination experiment which is in the condition of $8 \mathrm{~h}$ aeration and different $\mathrm{pH}$ value, we can see modified lignin flocculent effect is different and the settlement of COD lower level is not the same. After the aeration the $\mathrm{pH}$ of furfural wastewater rise from 4 to approximately 7 , and there is a settlement in the solution, then add flocculants and auxiliary sorbents, a large number of floce sedrmation will separate out from solution. From graph 2.3 we can see the biggest decline of COD occurs in the $\mathrm{pH}-9$. Because the condensation reaction occurs under this condition and molecular weight increase ceaselessly 
to format the aerosol particles, in addition flocculation agent can play a good role of bridge to prompt to macromolecular flocculating sedimentation.

\subsection{Flocculant for COD removal rate of consumption}

Taking $250 \mathrm{ml}$ fresh furfural liquid; $8 \mathrm{~h}$ aeration, adjust it to $\mathrm{pH}-9$. the graph 2.4 shows the change of furfural wastewater COD after adding the Modified lignin natural organic polymer flocculant and auxiliary adsorbent. As we can see from the graph when the amount of flocculants is bigger than $2 \mathrm{~g}$, the COD removal rate of furfural wastewater will be more than $50 \%$, if we increase the amount of flocculants; the COD removal rate will reach the highest rate of $60 \%$, the COD removal rate increase. Therefore, in the processing of dealing with furfural wastewater, the advisable dosing quantity of modified lignin flocculants is $8 \mathrm{~g} / \mathrm{L}$.

\section{Conclusion}

Irradiation graft copolymerization method is a kind of green chemical processing method. We can get the natural organic polymer flocculants when the modified lignin sulfonates has been processed, the flocculants is cheap price, non-toxic side effects, no secondary pollution and according with environmental protection requirement. the best condition of using This new type of flocculants is : $\mathrm{pH}-9$ of solution, dosage for $8 \mathrm{~g} / \mathrm{L}$, if it is combined with other technology, the COD removal rate can reach more than 50 percent in the furfural wastewater treatment engineering.

\section{References}

Fang GuiZhen, HeWeiHua \& Song ZhanQian. (2003). Cation flocculant lignin synthesis of salt and decoloring performance. Journal of Forest Chemical and Industrial, 23 (2): 37-42.

Liu Qianjun, ZhanHuaiYu, Liu mengru \& WuHong. (2003). Lignin and decoloring agents and flocculation properties of preparation and application. Hypertension, papermaking, science and technology, 22(6): 125-128.

Qiu XueQing, LouHongMing. (1999). Wood grain water treatment by namics. Environmental protection, 6: 45-47.

R. B. Phillips, W. Brown \& V. Stannett. (1972). The graft copolymerization of styrene and lignin. II. Kraft softwood lignin. Journal of Applied Polymer Science, 16(1): 1-14.

R. B. Phillips, W. Brown \& V. Stannett. (1973). The graft copolymerization of styrene and lignin. III. Chain transfer reactions of lignin and lignin model compounds. Journal of Applied Polymer Science, 17(2): 443-451.

Zhan HuaiYu. (2005). chemistry and physics. Cellulose. Beijing: science press: 226.
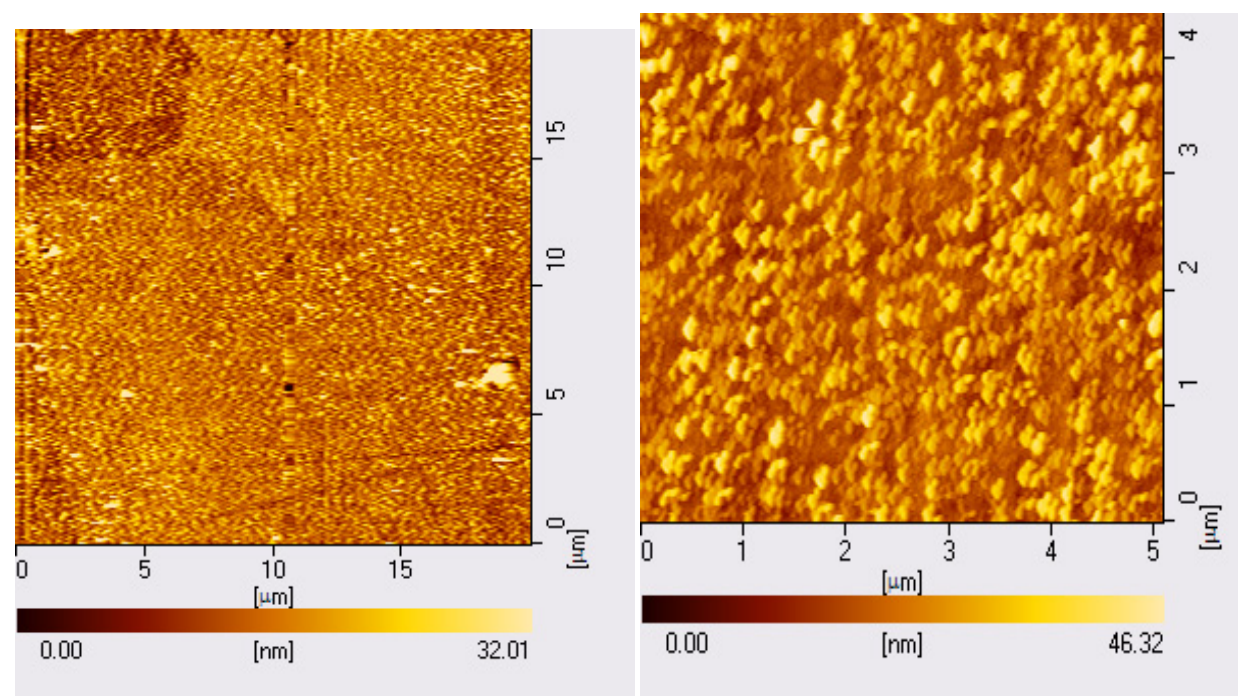

a. Before the modified lignin surface morphology b. Grafted modified lignin surface morphology

Figure 2.1 Modified lignin sulfonate membrane surface morphology 


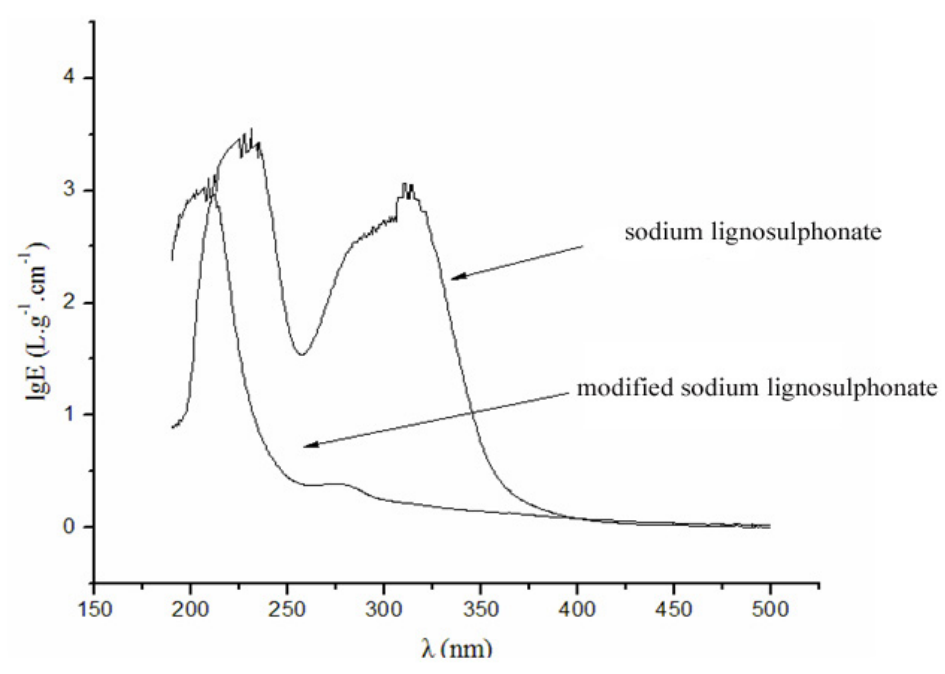

Figure 2.2 Modified lignin sulfonate up spectra

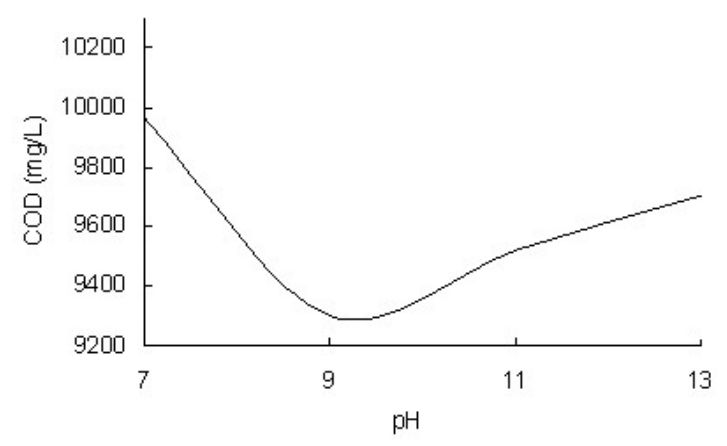

Figure 2.3 of furfural wastewater $\mathrm{pH}$ value of COD removal

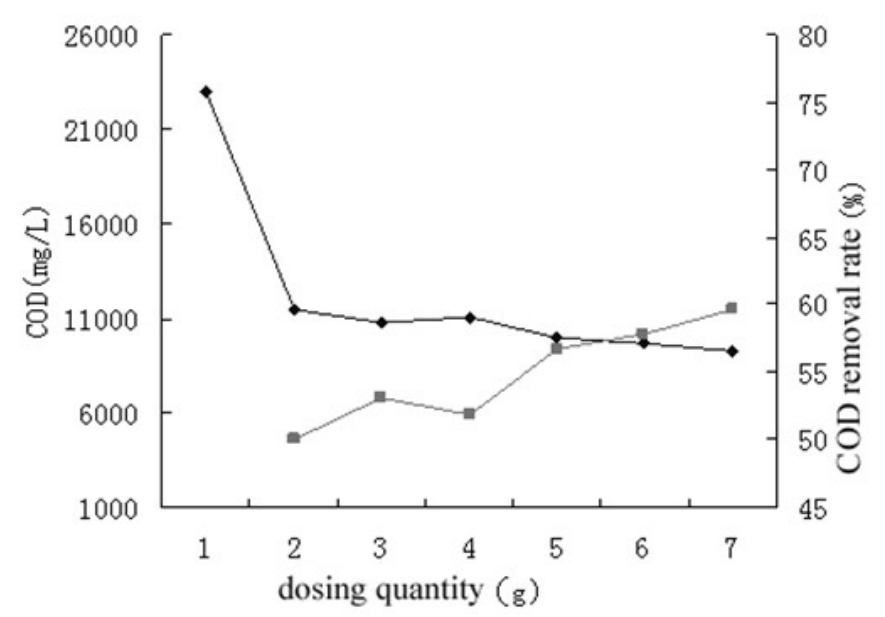

Figure 2.4 modified lignin flocculant dosing quantity of COD removal 